\title{
Response Characteristics of Looseness-Rubbing Coupling Fault in Rotor-Sliding Bearing System
}

\author{
Yang Liu, Zengyuan Xue, Lei Jia, Tuo Shi, and Hui Ma \\ School of Mechanical Engineering and Automation, Northeastern University, Shenyang, Liaoning 110819, China \\ Correspondence should be addressed to Yang Liu; liuyang1982@mail.neu.edu.cn
}

Received 26 August 2017; Accepted 9 November 2017; Published 5 December 2017

Academic Editor: Yuri Vladimirovich Mikhlin

Copyright (C) 2017 Yang Liu et al. This is an open access article distributed under the Creative Commons Attribution License, which permits unrestricted use, distribution, and reproduction in any medium, provided the original work is properly cited.

\begin{abstract}
For the diagnosis of looseness-rubbing coupling fault of rotor-sliding bearing system caused by bolt looseness fault, the mechanical model and finite element model of dual-disc rotor system with looseness-rubbing coupling fault are established based on the nonlinear finite element method, nonlinear oil film force, looseness stiffness model, and Hertz contact theory. With the augmented Lagrange method, contact constraint conditions are dealt with to ensure that the rotary disk and casing contact with each other meeting boundary penetrating depth within the prescribed tolerance range. And then the dynamics characteristics of the health rotor system supported by sliding bearing are studied. Combined with experimental study and simulation analysis, it is found that the looseness-rubbing coupling fault is often characterized by rubbing fault, the lower part of the time-domain fluctuated shape is denser, while the upper part is relatively loose, and multiple nested half ellipse is shown in orbit diagram. Because of the loosing stiffness and rubbing force, the phenomenon of unstable oil film is depressed. The appearance of the first- and second-order oil film oscillation phenomenon is delayed. It could be used as a theoretical basis for diagnosing looseness-rubbing coupling fault of rotor-sliding bearing system.
\end{abstract}

\section{Introduction}

Oil film oscillation is a common phenomenon in the rotorsliding bearing system. The oil film oscillation in the rotating machinery makes the shafting amplitude increase rapidly, which easily initiates the bolt looseness between the bearing chock and the foundation, and furthermore initiates collision and friction. It leads to a periodical rubbing phenomenon, which directly influences the safe operation of unit. Therefore, it has an important significance to research the complicated dynamics characteristic and its evolution regulation of the looseness-rubbing coupling fault of rotor-sliding bearing system to promote the stability and safety of the rotating machinery.

In recent years, the research on the looseness-rubbing coupling fault and the system instability problem which is caused by oil film oscillation is always an important subject which the scholars pay attention to. In order to simulate the nonlinearity of the sliding bearing, some nonlinear oil film force models have been proposed, such as Capone $\mathrm{G}[1,2]$. The Muszynska model [3-5] was introduced as a simple model of nonlinear fluid dynamic forces generated in bearings as well as in seals based on the results of a series of experiments. A parameter called the fluid average circumferential velocity ratio is used to describe the characteristic of the fluid motion as a whole. The model has been used to analyze antiswirl to prevent rotor/seal instability correctly and effectively. de Castro et al. [6] researched the system instability threshold influenced by the unbalance, rotor arrangement form, and bearing parameters. Jing et al. [7] use the oil film force which is obtained from bearing theory to model a continuum rotor-bearing system by the finite element method. Based on Capone model, Adiletta et al. [8] analyzed the possible chaotic motions resulting from the nonlinear response of bearings. Ding and Leung [9] considered an isotropic flexible shaft which is activated by nonlinear fluid-induced forces generated from oil-lubricated journal bearings and hydrodynamic seal.

Considering the coupled effect of the nonlinear oil film force and fault force such as looseness or rubbing fault, many researchers studied the dynamic behaviors of the rotorbearing system. The dynamic behavior in the rubbing of the rotor and stator based on the oil film force of the short 
bearing is researched by Chu and Zhang [10]. After the analysis of the research, it is found to be an obvious bifurcation and chaotic phenomenon. Liu et al. [11] study the stability of the periodic motion on the looseness-rubbing coupling fault of rotor-sliding bearing system and analyze the change of the bifurcation in different parameter regions of the system. Despite the response feature research of the rotor system in the looseness-rubbing coupling fault, Chen [12] finds that looseness fault can lead the amplitude of the system to increase rapidly and chaotic phenomenon appears. Muszynska and Goldman [13] study the nonlinear dynamic behavior in the rotor system of the base-looseness and rotor-stator rubbing fault and combine the vibration characteristics of the rotor system to complete the relevant experiment. Su et al. [14] use the model of short bearing with unsteady oil film force to study the random response problem of the rotor system where rubbing and support looseness exist. Luo et al. [15] study the periodic motion of the dual-span rotor with the looseness-rubbing coupling fault and point out that the response of the rotor system mainly consists of chaotic motion. Xiang et al. [16] used dynamics theory to establish the mathematical model of an asymmetric double-disc rotor-bearing system which was considering nonlinear oil film force and rub-impact force and pointed out that the phenomenon of the simulation would have a considerable value as diagnostic tools in settling oil film instability. Hu et al. [17] designed a dedicated experimental setup to validate the interaction between rubs and oil film vibration and put forward a model considering nonlinear rub-impact forces and nonlinear oil film forces to analyze the complicated nonlinear vibrations of the rotor-bearing system. The research discloses the worthy energy exchange phenomenon of multifault system and presents the nonlinear dynamical characteristics of the interaction between rubimpact and oil film instability for such a rotor-bearing system.

The dynamic finite element model of dual-disc and onespan rotor system with looseness-rubbing coupling fault is supported by oil film force, and based on the looseness stiffness model theory, Hertz contact theory, and the oil film force model of the short bearing, the model is established. And then the research has been conducted to deal with the contact constraint condition through the method of augmented Lagrange to make sure that the permeation depth of the boundary meets prescriptive tolerance range when the disc and the casing contact with each other. Furthermore, by the Newmark- $\beta$ numerical iteration solution method which is combined with the Newton-Raphson numerical iteration solution method, the excitation response result on the corresponding nodes of the fault rotor system is got. In the meanwhile, the relevant characteristics in the periodic and aperiodic motion of the looseness-rubbing coupling fault of rotor-sliding bearing system are analyzed with experimental study and simulation analysis. The research result represents that the looseness-rubbing coupling fault is often characterized by rubbing fault. And its frequency components are relatively rich. The rotation frequency is as the main frequency for a long time. The lower part of the timedomain fluctuated shape is denser, while the upper part is relatively loose, and multiple nested half ellipse is shown in

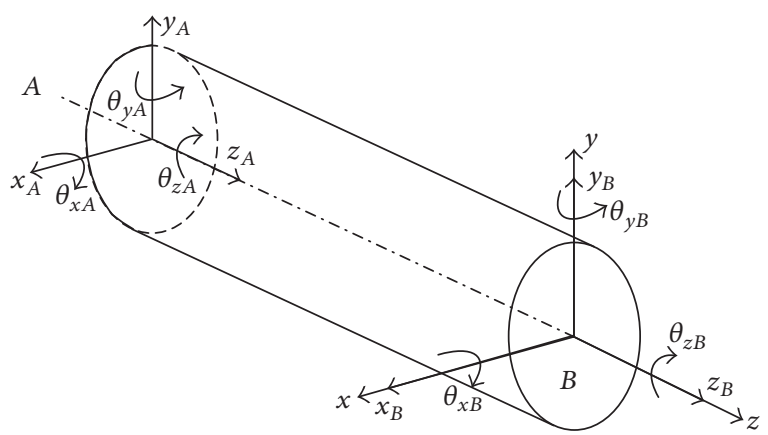

FIgURE 1: Timoshenko beam element.

orbit diagram. This could be used as a theoretical basis for diagnosing looseness-rubbing coupling fault of rotor-sliding bearing system.

\section{Dynamic Model of Rotor-Bearing System with the Fault}

The looseness-rubbing process of the rotor system which is supported by the oil film force is very complex. Because it is the main problem, the following assumption should be defined: (1) the shaft segment of the rotor system is simulated with Timoshenko beam element and only used two nodes (A and B) of end face in the beam element. In the meanwhile, the rotor system neglects the deformation on the axis direction and torsion deformation. Figure 1 is the schematic diagram of the shaft segment; (2) the oil film force from the sliding bearing uses the short bearing oil film force model which is in the literature [18]. And the effect of base loosening on oil film force is neglected; (3) only the basic elastic on the looseness direction ( $y$ direction) is considered.

Nodes A and B in the Timoshenko beam element each have six DOF. $x_{A}, y_{A}, z_{A}, \theta_{x A}, \theta_{y A}, \theta_{z A}$ are the displacement and angle of the $x, y, z$ direction on the node $A$; $x_{B}, y_{B}, z_{B}, \theta_{x B}, \theta_{y B}, \theta_{z B}$ are the displacement and angle of the $x, y, z$ direction on the node B. The DOF of the Timoshenko beam element range as $\mathbf{u}=\left[x_{A}, y_{A}, z_{A}, \theta_{x A}, \theta_{y A}, \theta_{z A}\right.$, $\left.x_{B}, y_{B}, z_{B}, \theta_{x B}, \theta_{y B}, \theta_{z B}\right]^{\mathrm{T}}$.

As shown in Figure 2, the rotor system with loosenessrubbing coupling fault supported by the sliding bearing has 26 nodes in all and divided into 24 shaft segments. To simulate the looseness fault of the support, one node which is named 26 is especially added. It is supported by the oil film force between node 26 and node 25 . A segmentation linear springdamper element is used to simulate between node 26 and the foundation and its equivalent loosening stiffness is shown in (2). The diameters of the two disks are $60 \mathrm{~mm}$. Their location is between shafts 7 and 18 . The left bearing and right bearing are, respectively, on node 1 and node 25 . The main parameters of the shaft segment are shown in Table 1.

2.1. The Model of Loosening Stiffness. As shown in Figure 3, the tension stiffness of the loosening bolt is $k_{f 1}$. The tension stiffness of the nonloosening bolt is $k_{f 2}$. The stiffness of 


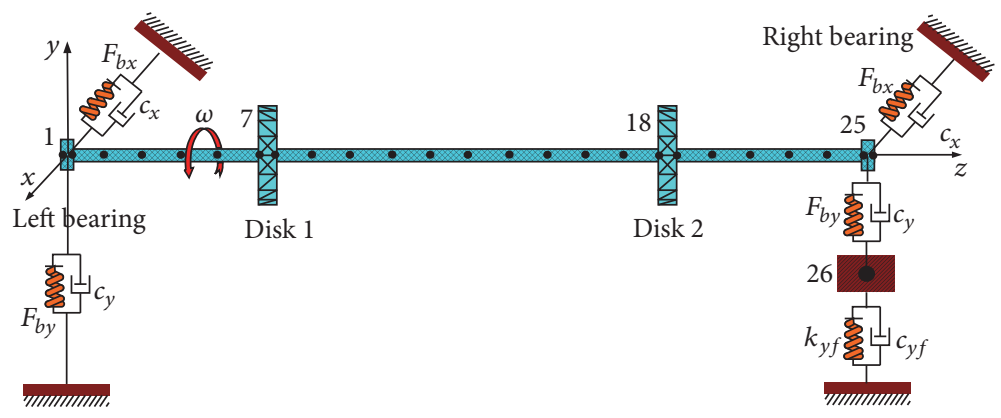

Figure 2: Dynamic model of fault rotor system.

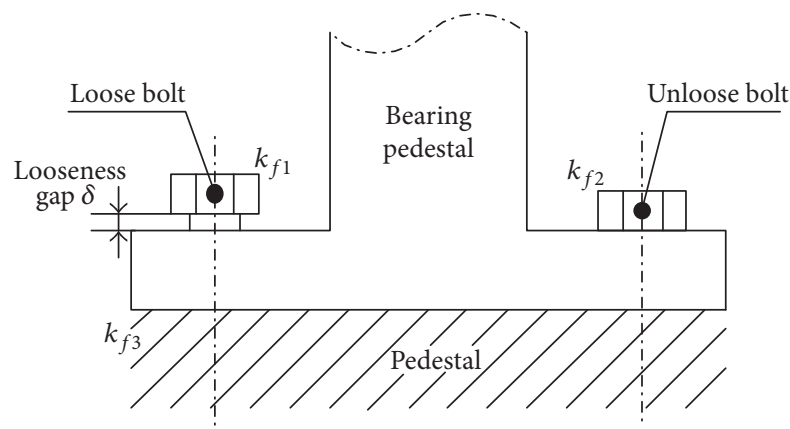

FIGURE 3: The schematic diagram of pedestal looseness.

TABLE 1: The main parameters of rotor system shaft segment.

\begin{tabular}{lcccccccccccc}
\hline Property & \multicolumn{11}{c}{ Data } \\
\hline Shaft segment & 1 & 2 & 3 & 4 & 5 & 6 & 7 & 8 & 9 & 10 & 11 & 12 \\
Length/mm & 10 & 40 & 40 & 40 & 40 & 40 & 20 & 40 & 40 & 40 & 40 & 40 \\
Diameter/mm & 20 & 20 & 20 & 20 & 20 & 20 & 60 & 20 & 20 & 20 & 20 & 20 \\
\hline Shaft segment & 13 & 14 & 15 & 16 & 17 & 18 & 19 & 20 & 21 & 22 & 23 & 24 \\
Length/mm & 40 & 40 & 40 & 40 & 40 & 20 & 40 & 40 & 40 & 40 & 40 & 10 \\
Diameter/mm & 20 & 20 & 20 & 20 & 20 & 60 & 20 & 20 & 20 & 20 & 20 & 20
\end{tabular}

the foundation (ground) is $k_{f 3}$. When the rotor has a small vibration on the radial direction, the bearing pedestal and the foundation contact with each other. The foundation supports the bearing pedestal. Then the equivalent stiffness of the loosening side is the stiffness of the foundation, $k_{f}=k_{f 3}$. Because of the phenomenon of the bolt looseness, when amplitude is large, the bearing pedestal could get rid of the constraint of the loosening bolt and be separated from the foundation temporarily. At this time, the nonloosening bolt is in the condition of elastic deformation. The equivalent stiffness of the loosening side is $k_{f}=k_{f 2}$; if the displacement of the bearing pedestal is larger than the loosening bolt gap, then the equivalent stiffness $k_{f} \approx k_{f 1}+k_{f 2}$. So the equivalent stiffness of the loosening [19] side can be summarized as follows:

$$
k_{f}= \begin{cases}k_{f 1}+k_{f 2}, & (y>\delta), \\ k_{f 2}, & (0 \leq y \leq \delta), \\ k_{f 3}, & (y<0) .\end{cases}
$$

An assumption is given that the foundation stiffness $k_{f 3}$ is approximate with the sum of the tension stiffness of all the loosening bolt. $k_{f 3} \approx k_{f 1}+k_{f 2}$. Equation (1) can be simplified to

$$
k_{f}= \begin{cases}k_{f 2}, & (0 \leq y \leq \delta), \\ k_{f 3}, & \text { the rest. }\end{cases}
$$

2.2. The Rubbing Force Model Based on Contact Judgment. For the safety, the disc (or vane) of the rotor system should be protected by the casing. It is assumed that the rotary disk 1 is protected by the casing. When the system is at rest, the radius clearance between the rotor and the casing (stator) is shown in Figure 4. It is assumed that the cross section of the rotary disk is always keeping in the plane xoy. $O$ is the center of the rotor. $o_{1}$ is still the center of the stator. $\omega$ is the rotational velocity. This paper completes the contact detection based on the Kuhn-Tucker condition and deals with the contact constraint condition through the method of augmented Lagrange [20] to make sure that the permeation depth of the boundary meets prescriptive tolerance range when the rotary disk and the casing contact with each other. So the rubbing force $F_{\text {rub }}$ can be divided into the rubbing force on the normal direction $\left(F_{r}\right)$ and the tangential direction $\left(F_{t}\right)$. Its expression is

$$
\begin{aligned}
& F_{r}=(r-c) k_{r}+\lambda_{r}^{(i+1)}, \\
& F_{t}=f F_{r},
\end{aligned}
$$

$$
(r \geq c)
$$

where $k_{r}$ is the rubbing stiffness; $f$ is the friction coefficient at the friction point; $c$ is the radius gap between the rotor and stator; $r$ is the normal direction of the rotor; $\lambda_{r}^{(i+1)}$ is the 


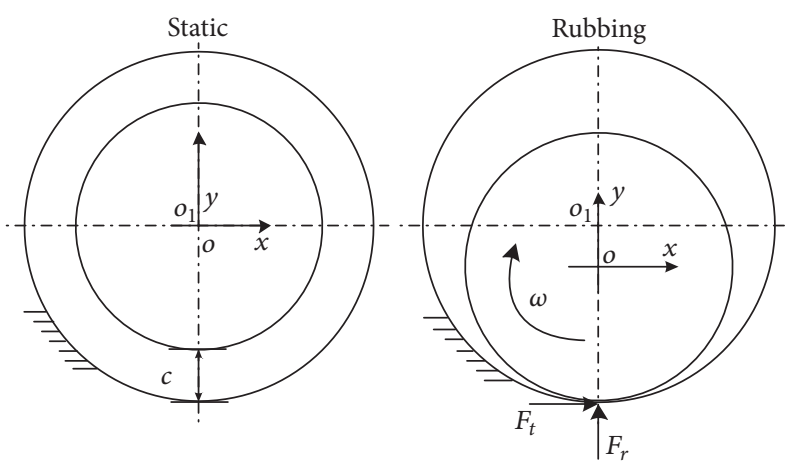

Figure 4: The model of the rubbing force.

Lagrange multiplier in the iteration process on the $i+1$ step, and its expression is

$$
\lambda_{r}^{(i+1)}= \begin{cases}\lambda_{r}^{(i)}+k_{r} \delta_{r}, & \left|\delta_{r}\right| \geq \varsigma \\ \lambda_{r}^{(i)}, & \left|\delta_{r}\right|<\varsigma\end{cases}
$$

where $\delta_{r}=r-c$; $\varsigma$ is the allowed permeation tolerance value; when the value is over $\varsigma$, the rubbing force on the normal direction $F_{r}$ expands through the Lagrange multiplier $\lambda_{r}^{(i+1)}$ and iterates continuously until the permeation value is smaller than the maximum allowed value $\varsigma$.

In the xoy reference frame, the rubbing force along the $x$ direction and $y$ direction can be expressed as

$$
\left\{\begin{array}{c}
F_{r x} \\
F_{r y}
\end{array}\right\}=-H\left(\delta_{r}\right) \delta_{r} \frac{\delta_{r} k_{r}+\lambda_{r}^{i+1}}{r}\left(\begin{array}{cc}
1 & -f \\
f & 1
\end{array}\right)\left\{\begin{array}{l}
x_{1} \\
y_{1}
\end{array}\right\},
$$

where $x_{1}$ is the displacement of the rotor axis on the $x$ and $y$ direction. $y_{1}$ is the displacement of the rotor axis on the $y$ direction. $H\left(\delta_{r}\right)$ is the step function.

$$
H\left(\delta_{r}\right)= \begin{cases}1, & \delta_{r} \geq 0 \\ 0, & \delta_{r}<0\end{cases}
$$

The meaning of the expression above is that while $r<$ $c$, the rubbing phenomenon does not happen and then the rubbing force is 0 . However $r \geq c$, the rubbing phenomenon happens and the rubbing force appears.

2.3. The Finite Element Model of the Fault Rotor System. As known above, under the support of the sliding bearing, the bolt looseness and the rotary disk rubbing with the blades are considered in the rotor-bearing system of which finite element model is shown below.

$$
\mathbf{M} \ddot{\mathbf{u}}+(\mathbf{C}+\mathbf{G}) \dot{\mathbf{u}}+\mathbf{K u}=\mathbf{F}_{\mathbf{e}}+\mathbf{F}_{\mathbf{b}}+\mathbf{F}_{\text {rub }},
$$

where $\mathbf{M}, \mathbf{G}, \mathbf{C}, \mathbf{K}, \mathbf{F}_{\mathbf{e}}, \mathbf{F}_{\mathbf{b}}, \mathbf{F}_{\text {rub }}$ are, respectively, the mass matrix, gyroscope matrix, damping matrix, stiffness matrix, the vector of the unbalance force, the vector of the oil film force, and the vector of the rubbing force. Because the normal deformation on the node of the model is much larger than the deformation on the axial direction and the magnitude has a huge difference, neglect the displacement vector on the axial direction and after the torsion deformation. The displacement vector $\mathbf{u}=\left[x_{A}, y_{A}, \theta_{x A}, \theta_{y A}, x_{B}, y_{B}, \theta_{x B}, \theta_{y B}\right]^{\mathrm{T}}$. The damping in the model uses the expression of the Rayleigh damping.

$$
\mathbf{C}=\alpha \mathbf{M}+\beta \mathbf{K}
$$

where $\alpha$ is quality proportion coefficient and $\beta$ is stiffness proportion coefficient

$$
\begin{aligned}
& \alpha=\frac{2\left(\xi_{2} / \omega_{n 2}-\xi_{1} / \omega_{n 1}\right)}{1 / \omega_{n 2}^{2}-1 / \omega_{n 1}^{2}}, \\
& \beta=\frac{2\left(\xi_{2} \omega_{n 2}-\xi_{1} \omega_{n 1}\right)}{\omega_{n 2}^{2}-\omega_{n 1}^{2}},
\end{aligned}
$$

$\omega_{n 1}$ is the first-order critical speed, $\omega_{n 2}$ is the second-order critical speed, $\xi_{1}$ is the first-order modal damping ratio; $\xi_{2}$ is the second-order modal damping ratio.

The two support bearings use the model of the short bearing oil film force and then get a corresponding promotion and normalization processing. $\mathbf{F}_{\mathbf{b x}}$ and $\mathbf{F}_{\mathbf{b y}}$ are, respectively, the nonlinear oil film force $\left(\mathbf{F}_{\mathbf{b}}\right)$ of the bearing on the $x, y$ direction vector, and its expression is

$$
\begin{aligned}
& {\left[\begin{array}{l}
\mathbf{F}_{\mathbf{b x}} \\
\mathbf{F}_{\mathbf{b y}}
\end{array}\right]=\sigma\left[\begin{array}{l}
\mathbf{f}_{\mathbf{b x}} \\
\mathbf{f}_{\mathbf{b y}}
\end{array}\right],} \\
& {\left[\begin{array}{l}
f_{b x} \\
f_{b y}
\end{array}\right]=\frac{\left[(\tilde{x}-2 \dot{\tilde{y}})^{2}+(\tilde{y}+2 \dot{\tilde{x}})^{2}\right]^{1 / 2}}{1-\tilde{x}^{2}-\tilde{y}^{2}}} \\
& \quad \times\left[\begin{array}{l}
3 \tilde{x} V(\tilde{x}, \tilde{y}, \alpha)-\sin \alpha G(\tilde{x}, \tilde{y}, \alpha)-2 \cos \alpha S(\tilde{x}, \tilde{y}, \alpha) \\
3 \tilde{y} V(\tilde{x}, \tilde{y}, \alpha)+\cos \alpha G(\tilde{x}, \tilde{y}, \alpha)-2 \sin \alpha S(\tilde{x}, \tilde{y}, \alpha)
\end{array}\right],
\end{aligned}
$$

where

$$
\begin{aligned}
& \mathbf{V}(\widetilde{\mathbf{x}}, \widetilde{\mathbf{y}}, \alpha)=\frac{2+(\widetilde{\mathbf{y}} \cos \alpha-\widetilde{\mathbf{x}} \sin \alpha) \mathbf{G}(\widetilde{\mathbf{x}}, \widetilde{\mathbf{y}}, \alpha)}{1-\widetilde{\mathbf{x}}^{2}-\widetilde{\mathbf{y}}^{2}}, \\
& \mathbf{S}(\widetilde{\mathbf{x}}, \widetilde{\mathbf{y}}, \alpha)=\frac{\widetilde{\mathbf{x}} \cos \alpha+\widetilde{\mathbf{y}} \sin \alpha}{1-(\widetilde{\mathbf{x}} \cos \alpha+\widetilde{\mathbf{y}} \sin \alpha)^{2}}, \\
& G(\tilde{x}, \tilde{y}, \alpha)=\frac{2}{\left(1-\tilde{x}^{2}-\tilde{y}^{2}\right)^{1 / 2}}\left[\frac{\pi}{2}\right. \\
& \left.+\arctan \frac{\tilde{y} \cdot \cos \alpha-\tilde{x} \cdot \sin \alpha}{\left(1-\tilde{x}^{2}-\tilde{y}^{2}\right)^{1 / 2}}\right] \\
& \alpha=\arctan \left(\frac{\tilde{y}+2 \dot{\tilde{x}}}{\tilde{x}-2 \dot{\tilde{y}}}\right)-\frac{\pi}{2} \operatorname{sign}\left(\frac{\tilde{y}+2 \dot{\tilde{x}}}{\tilde{x}-2 \dot{\tilde{y}}}\right)-\frac{\pi}{2} \\
& \cdot \operatorname{sign}(\tilde{y}+2 \dot{\tilde{x}})
\end{aligned}
$$

where $\sigma$ is the Sommerfeld coefficient; and its expression is

$$
\sigma=\eta \omega \frac{\mathbf{D}}{2} \mathbf{L}\left(\frac{\mathbf{D}}{2 \tau}\right)^{2}\left(\frac{\mathbf{L}}{\mathbf{D}}\right)^{2},
$$


where $L$ is bearing width; $\eta$ is oil viscosity; $D$ is bearing diameter; $\tau$ is oil film gap.

The Newmark- $\beta$ numerical algorithm combined with the Newton-Raphson iteration method has been used to solve the nonlinear differential equation (7) which is established formerly. And then the vibrating response data on the relevant nodes of the finite element model are got to analyze the fault of rotor system.

\section{Dynamics Characteristic Analysis of Coupling Fault of Rotor System}

The main parameters of the looseness-rubbing coupling fault of rotor system are shown below. The shaft diameter is $20 \mathrm{~mm}$, the shaft length is $860 \mathrm{~mm}$, the disc diameter is $60 \mathrm{~mm}$, the disc thickness is $20 \mathrm{~mm}$, the unbalance quality is $156 e-$ $6 \mathrm{~kg} \cdot \mathrm{m}$, the material density $\rho=7850 \mathrm{~kg} / \mathrm{m}^{3}$, the elastic modulus $E=2.06 \times 10^{11} \mathrm{~Pa}$, the Poisson ratio $v=0.3$, the rubbing gap is $u=200 \mu \mathrm{m}$, the rubbing stiffness $k_{r}=2 \times 10^{7} \mathrm{~N} / \mathrm{m}$, the friction coefficient $f=0.3$, and the left and right bearing use the same parameter of the oil film force. The bearing width $L=10 \mathrm{~mm}$, the viscosity $\eta=0.04 \mathrm{~Pa} \cdot \mathrm{s}$, the bearing diameter $D=20 \mathrm{~mm}$, the oil film gap $c=0.3 \mathrm{~mm}$, the bearing damping $d=2 \times 10^{3} \mathrm{~N} /(\mathrm{m} / \mathrm{s})$, and the eccentric magnitude is $0.1 \mathrm{~mm}$.

\subsection{The Influence of the Oil Film Force on the Dynamics} Characteristic of Rotor System. In the role of oil film force, the system will show oil film instability phenomenon [2125]. To clearly observe the dynamics characteristic of rotor system under the oil film force, then specially draw the threedimensional spectrum on the right neck (node 25) of the rotor system without fault, shown in Figure 5 . When the speed is $0 \leq \omega<2830 \mathrm{r} / \mathrm{min}$, the rotor system represents a synchronous positive precession. And then, there are twice frequency $2 f_{r}$, three times frequency $3 f_{r}$, and other higherorder times frequency with little peak besides the rotation frequency $f_{r}$. While the speed is $2830 \leq \omega \leq 3460 \mathrm{r} / \mathrm{min}$, the eddy turbulence of the rotor system appears, and the frequency is nearly half the rotation frequency $f_{r}$. When the rotational speed continues to increase to $3460<\omega<$ $4810 \mathrm{r} / \mathrm{min}$, the oil film instability of the rotor is suppressed by the rapid increase in the frequency component, and the oil film vortex phenomenon disappears temporarily. There are $f_{r}, 2 f_{r}$, and $3 f_{r}$ on the spectrum at this time. When the speed is $\omega \geq 4810 \mathrm{r} / \mathrm{min}$, the first-order oil film oscillation phenomenon appears, and the frequency is $27.5 \mathrm{~Hz}$.

Through the evolution process analysis of the dynamics characteristic of rotor system supported by oil film force, and comparing the simulation result with the Muszynska experiment result in the literature [18], it is shown that the simulation code of the oil film force in this paper can fit the experiment result in the literature [18] well to some extent and verify the accuracy of the simulation code of the oil film force.

3.2. Dynamics Characteristic Analysis of Rotor System with Fault. The frequency spectrogram of rotor system with looseness-rubbing coupling fault under the nonlinear oil film force is shown in Figure 6. From Figure 6(a), while the speed

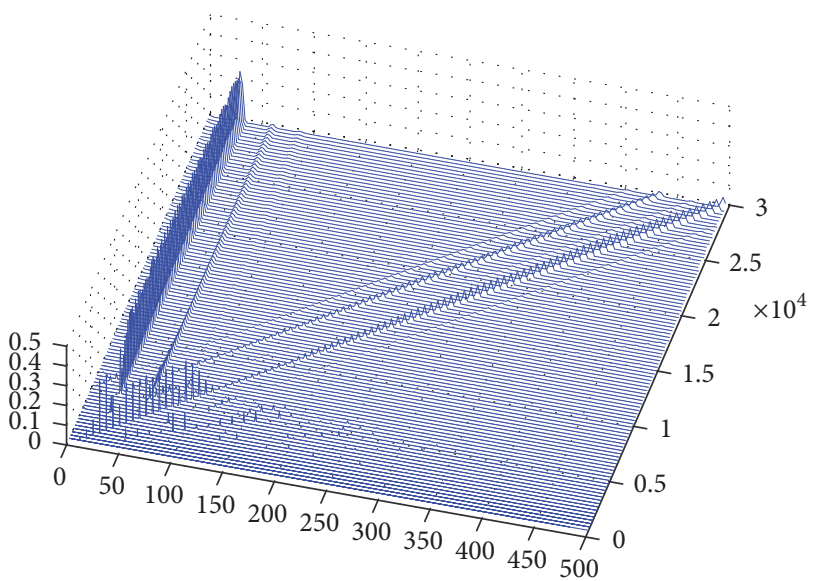

FIGURE 5: The spectrum waterfall of the rotor system without fault.

is a little low, the system under the oil film force shows some high frequency components which are not obvious. It is mainly the twice frequency $2 f_{r}$ and three times frequency $3 f_{r}$. While the speed is above $4000 \mathrm{r} / \mathrm{min}$, the situation of the rotor-stator rubbing is gradually serious and the friction radian increases. Under the increasing rubbing force, the phenomenon of oil film oscillation is depressed and the main character of the system is the rubbing fault. In the meanwhile, because of the existence of the base loosing fault, the half frequency $1 / 2 f_{r}$, one-third frequency $1 / 3 f_{r}$, and even a quarter of frequency $1 / 4 f_{r}$ appear in the vibration signal. The rotating frequency amplitude gradually decreases instead. The low-order harmonic becomes the dominant frequency instead of the rotating frequency.

The radial runout can be caused by the base loosing fault of the rotor system, and the low frequency vibration can be excited. In the meanwhile, the vibration of multitimes fundamental frequency exists under the rubbing force and the oil film force. However, the twice-fundamental frequency is dominant. The more serious the system fault is, the larger the proportion the frequency division and frequency doubling occupy. By studying the peak trend in the vibrating frequency of the looseness-rubbing coupling fault of rotor system which has the representative half frequency $1 / 2 f_{r}$, the rotating frequency $f_{r}$, and the twice frequency $2 f_{r}$, it is helpful to judge the severity of the fault. Figure 6(b) is the peak curves spectrogram with the changing speed of the coupling fault system which has the half frequency $1 / 2 f_{r}$, the rotating frequency $f_{r}$, and the twice frequency $2 f_{r}$. Shown from the figure, while the speed is about $2500 \mathrm{r} / \mathrm{min}$, the peak of the rotating frequency $f_{r}$ and the twice frequency $2 f_{r}$ reach the highest point. ( $f_{r}$ is $8.233,2 f_{r}$ is 3.176$)$, and then there is no half frequency $1 / 2 f_{r}$. The rotating frequency is the dominant frequency. While the speed increases to $3500 \mathrm{r} / \mathrm{min}$, the half frequency $1 / 2 f_{r}$ appears. Its peak gradually surpasses the rotating frequency to be the dominant frequency and then reaches the top point $\left(1 / 2 f_{r}\right.$ is 6.953) near the speed of $4500 \mathrm{r} / \mathrm{min}$. Subsequently it begins to decrease and the peak of the half frequency $1 / 2 f_{r}$ trends to 0 while the speed interval is between $6000 \mathrm{r} / \mathrm{min}$ and $8500 \mathrm{r} / \mathrm{min}$. 


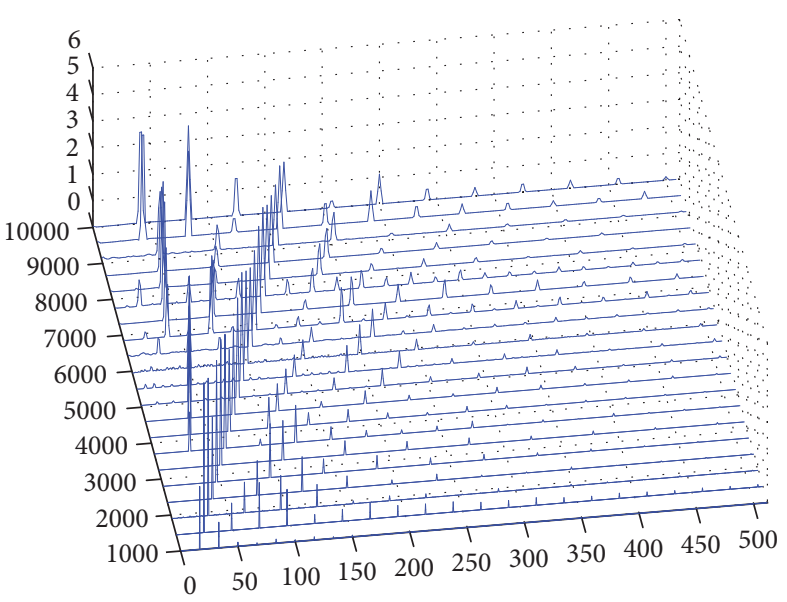

(a)

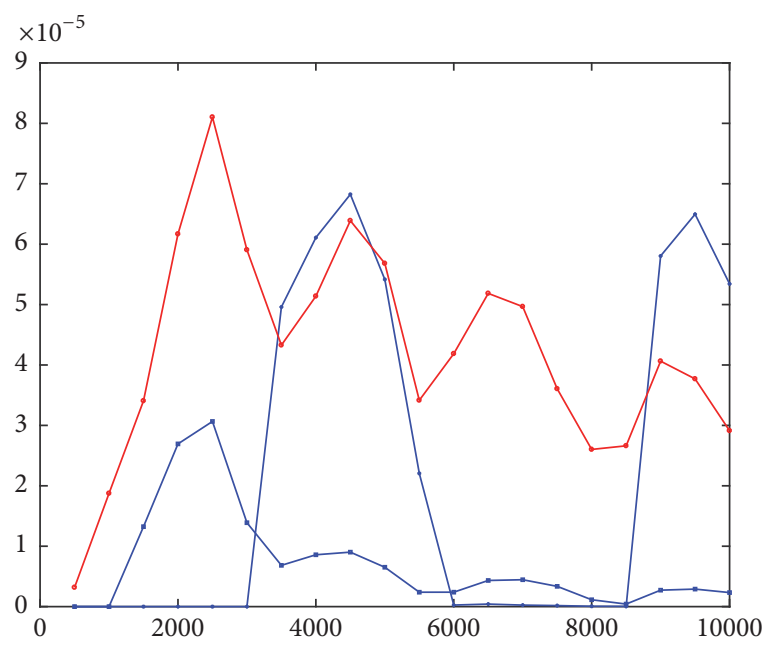

(b)

FIGURE 6: The frequency spectrogram of rotor system with coupling fault.

When the speed is above $8600 \mathrm{r} / \mathrm{min}$, the half frequency $1 / 2 f_{r}$ increases fast. And then the rotating frequency $f_{r}$ and the twice frequency $2 f_{r}$ enter the growing step; however they do not surpass the frequency division to be the dominant frequency. From the total trend of the every vibrating frequency peak, the trend of the rotating frequency $f_{r}$ and the twice frequency $2 f_{r}$ are nearly the same and are both the decreasing trend with increasing the speed. However, from the trend of the half frequency $1 / 2 f_{r}$, its speed quickly increases and surpasses the rotating frequency and the twice frequency on the speed interval $3000 \mathrm{r} / \mathrm{min}-6000 \mathrm{r} / \mathrm{min}$ and $8500 \mathrm{r} / \mathrm{min}-10000 \mathrm{r} / \mathrm{min}$. Combined with Figure $6(\mathrm{a})$, on the step of the $1 / 2 f_{r}$ peak (above $6000 \mathrm{r} / \mathrm{min}$ ) there are the $1 / 3$ harmonic and $1 / 4$ harmonic and their peaks are very large and surpass the rotating frequency. In total, the fault degree of rotor system is gradually serious with the speed increasing. The peaks of the rotating frequency and the twice frequency are gradually decreasing. A series of the frequency division harmonic gradually increases and its peak quickly increases. All the above illustrate that the constantly increasing speed leads to the rubbing fault, and then the system enters the step of coupling fault. The severity of the fault develops further.

It is assumed that the eccentric mass only has an effect on the two rotary disks, and the rubbing fault happens on the right of the rotary disk which is on rotary disk 2 . To distinguish the dynamics characteristic of looseness-rubbing coupling fault of rotor system under the support by oil film force, in the condition of foundation stiffness $k_{f 3}=1 \times$ $10^{9} \mathrm{~N} / \mathrm{m}$, the bolt tensile stiffness (loosing stiffness) $k_{f 2}=$ $8 \times 10^{5} \mathrm{~N} / \mathrm{m}$, and loosing gap $\delta=10 \mu \mathrm{m}$, Figures $7(\mathrm{a})-7(\mathrm{c})$ are the time-domain graph, orbit diagram, frequency spectrogram, and Poincare graph which are drawn by the steady response data on rotary disk 2 of the system with the speed $\omega$ being $3000 \mathrm{r} / \mathrm{min}, 4500 \mathrm{r} / \mathrm{min}$, and $8000 \mathrm{r} / \mathrm{min}$.

As shown in Figure 7, the time-domain waveforms of the rotor system produce distortion under the loosing stiffness and oil film force. And then the shape of time-domain gradually appears, and the lower part of the time-domain fluctuated shape is denser, while the upper part is relatively loose. The axis orbit is a semiellipse. With the speed increasing, the rotor-stator rubbing fault is gradually obvious. The axis orbit changes from a single semiellipse to many semiellipses which nest each other. Because of the influence on the eddy turbulence phenomenon, the twice frequency $2 f_{r}$ appears and then lots of frequency components appear. The half frequency $1 / 2 f_{r}$, the $1 / 3$ harmonic, $1 / 4$ harmonic, and $1 / 5$ harmonic appear in turn and the frequency division surpasses the rotation frequency to be the dominant frequency in the speed region. The Poincare graph gradually changes from one isolated point to two points with the speed increasing and then develops to five points. It unfolds the vibrating characteristic of frequency division of the system. The system is from periodic motion 1 to periodic motion 2 and then bifurcates to periodic motion 5 .

In total, because of the existence of the loosing fault in the rotor system under the support of the oil film force, the rubbing fault of the rotary disk and the casing is caused with the speed increasing, which leads to the fault degree of the rotor system gradually increased, a series of frequency division components are increasing, and the phenomenon of oil film oscillation is lagging.

3.3. Experiment Study of the Fault Rotor System. Build a fault rotor test-bed based on the simulation data above. Choose the portable analytic instrument BK3560D made in the B\&K company to acquire and analyze the data. The sampling frequency is $3.2 \times 10^{3} \mathrm{~Hz}$. To prevent the eddy current sensor from disturbing each other, the test points on the $x$ direction are $15 \mathrm{~mm}$ away from the test points on the $y$ direction. The position of the test point is nearest to the number 25 node of the rotor system in Figure 2, so admit that the displacement sensor is installed at node 25 (right bearing of the model). 

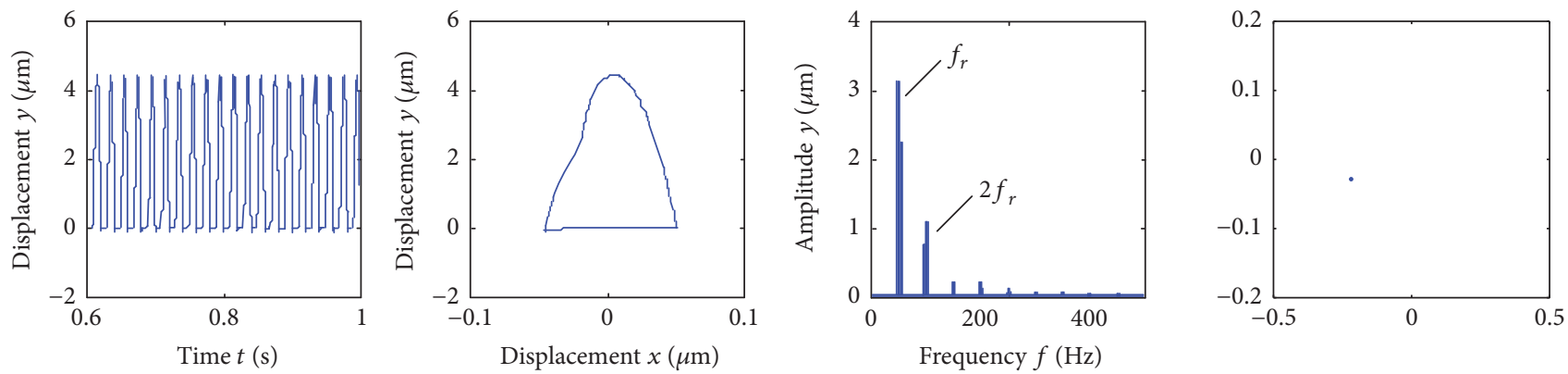

(a) Rotational speed $\omega=3000 \mathrm{r} / \mathrm{min}$
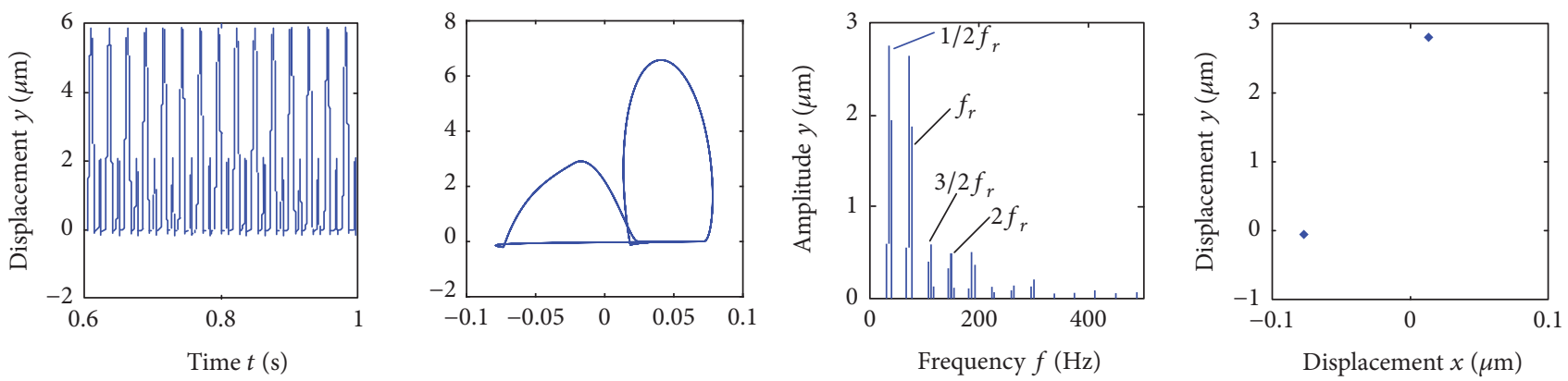

(b) Rotational speed $\omega=4500 \mathrm{r} / \mathrm{min}$
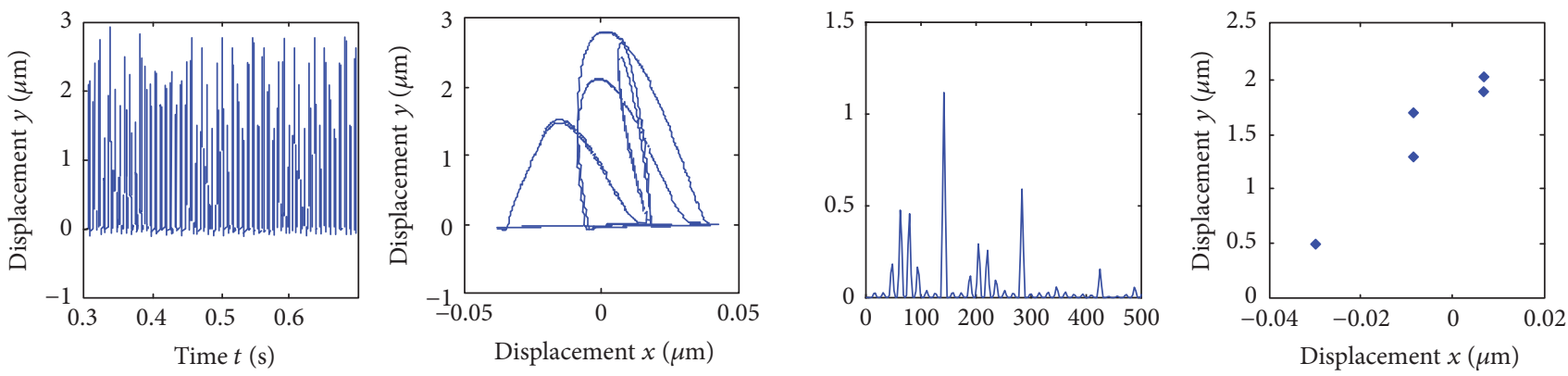

(c) Rotational speed $\omega=8000 \mathrm{r} / \mathrm{min}$

FIGURE 7: Time-domain graph, orbit diagram, frequency spectrogram, and Poincare graph of rotor system with different rotational speed.

Release the bolt on the bearing base a little, and then the loosing fault of the bolt appears. Pay attention to the loosing situation of the bolt to avoid the accident in the experiment. Choose the brass as the rubbing stator material and the C45 as the rotor material. The bearing type is made of oil-bearing graphite plain bearings. The rotor test-bed is shown in Figure 8.

Through the experiment, the three-dimensional frequency spectrum near the test point of the right bearing on the vertical direction ( $y$ direction) can be obtained. Shown in Figure 9, there is only the rotation frequency in the low speed region. With the speed increasing, because of the existence of looseness fault, high power frequency division begins to appear. The first-order oil film oscillation appears in the following speed region, and then the oil film oscillation disappears. With the speed continuously increasing, the first-order oil film oscillation happens. While the speed continuously increases, the peak of the first-order oil film oscillation temporarily reduces and the second-order oil film oscillation happens. In the meanwhile, because of the existence of the looseness-rubbing fault, the proportion of the vibrating energy occupied by the frequency division and frequency doubling also increases.

Contrasting the combined frequency characteristics in every speed region acquired by the simulation with the result by the experiment, the comparison result is shown in Table 2 . From the table we can see that the test results and simulation results in the overall evolution of the trend are basically the same.

The comparison of simulation and experiment shows that, in the simulation, the first- and second-order oil film oscillations occur after about $5000 \mathrm{r} / \mathrm{min}$. The frequency of first-order oil film oscillation gradually replaces the rotation frequency as the main frequency. At the same time, the relevant combination frequency also appears. However, in the experiment, due to external interference and the presence of noise signals, the frequency division appears earlier, and the peak of frequency becomes higher. Meanwhile, the first- and 


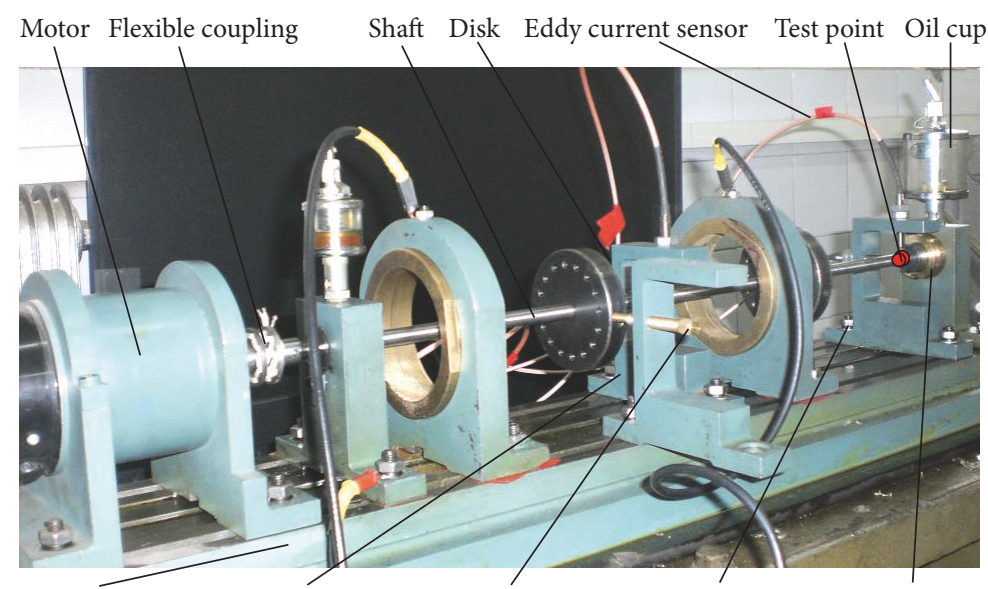

Pedestal Sensor holder Rubbing rod Loosening bolt Sliding bearing

FIGURE 8: The schematic diagram of the rotor test-bed with fault.

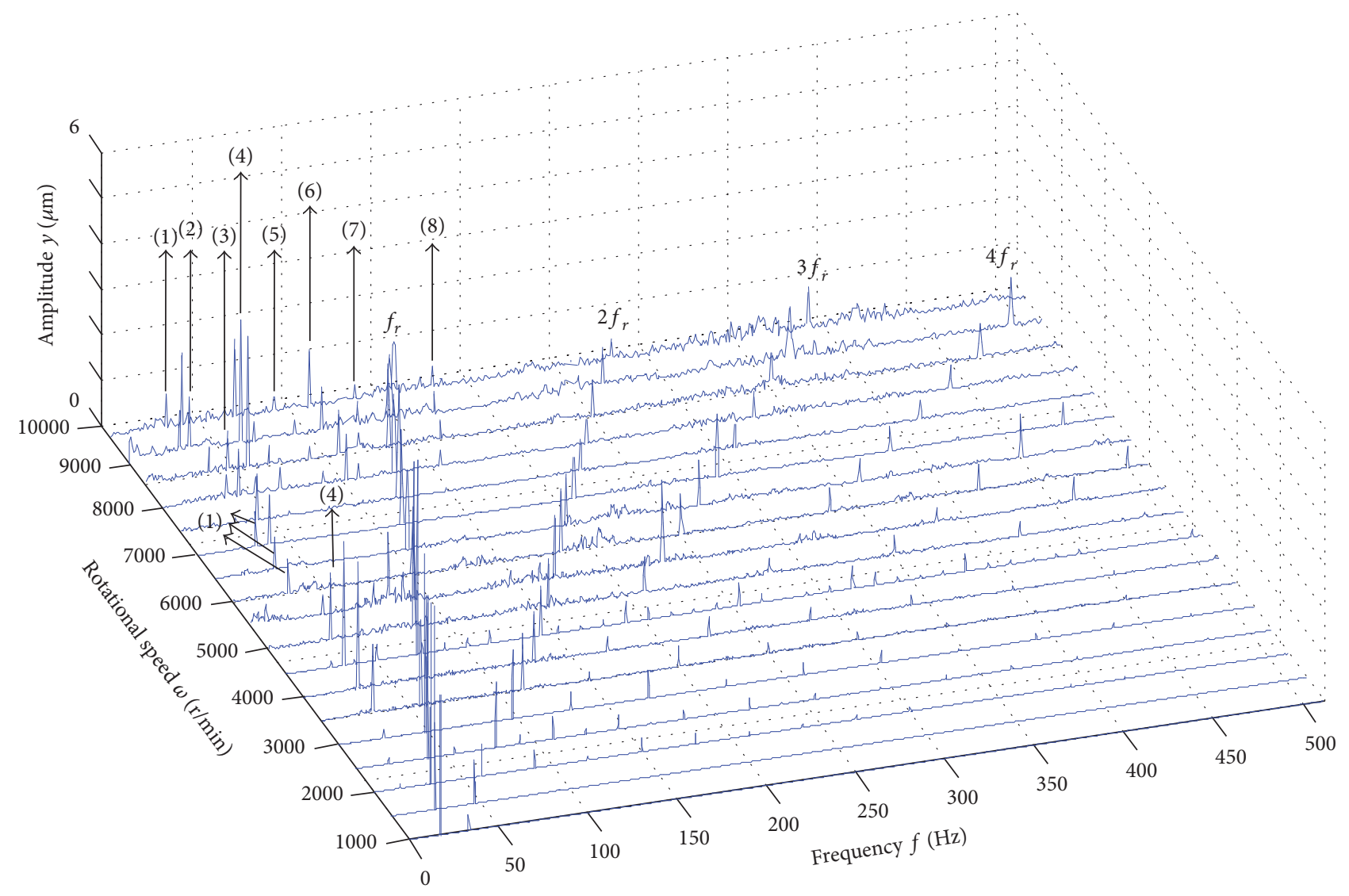
(1) $1 / 4 f_{r}$
(2) $f_{n 1}$
(5) $2 f_{n 1}$
(3) $1 / 3 f_{r}$
(6) $f_{n 2}$
(4) $1 / 2 f_{r}$
(7) $f_{r}-f_{n 1}$
(8) $f_{r}+f_{n 1}$

FIGURE 9: The frequency spectrum of the rotor test-bed with fault. 
TABLE 2: The combined frequency comparison of the simulation and the experiment.

\begin{tabular}{|c|c|c|}
\hline & Rotational speed/(r/min) & Frequency component/Hz \\
\hline \multirow{7}{*}{ Simulation } & $100 \leq \omega<990$ & $f_{r}$ \\
\hline & $1,000 \leq \omega \leq 2,750$ & $f_{r}, 2 f_{r}, 3 f_{r}$ \\
\hline & $2,750<\omega<5,930$ & $1 / 2 f_{r}, f_{r}, 3 / 2 f_{r}, 2 f_{r}, 5 / 2 f_{r}, 3 f_{r}$ \\
\hline & $5,930 \leq \omega \leq 7,980$ & $f_{n 1}, f_{r}-f_{n 1}, f_{r}, f_{r}+f_{n 1}, 3 / 2 f_{r}, 2 f_{r}, 5 / 2 f_{r}, 3 f_{r}$ \\
\hline & $7,980<\omega<8,460$ & $f_{n 1}, 1 / 3 f_{r}, f_{r}-f_{n 1}, 2 / 3 f_{r}, f_{r}, f_{r}+f_{n 1}, 4 / 3 f_{r}, 5 / 3 f_{r}, 2 f_{r}, 3 f_{r}, 1 / 3 f_{r}$ spectrum series, etc. \\
\hline & $8,460<\omega<9,520$ & $\begin{array}{c}1 / 3 f_{r}, 1 / 2 f_{r}, f_{r}-f_{n 1}, 2 / 3 f_{r}, f_{r}, f_{r}+f_{n 1}, 4 / 3 f_{r}, 3 / 2 f_{r}, 5 / 3 f_{r}, 2 f_{r}, 3 f_{r}, 1 / 3 f_{r} \text { spectrum series, } \\
1 / 2 f_{r} \text { spectrum series, etc. }\end{array}$ \\
\hline & $9,520<\omega<10,000$ & $\begin{array}{l}1 / 4 f_{r}, 1 / 3 f_{r}, 1 / 2 f_{r}, f_{r}-f_{n 1}, 2 / 3 f_{r}, 3 / 4 f_{r}, f_{r}, f_{r}+f_{n 1}, 5 / 4 f_{r}, 4 / 3 f_{r}, 3 / 2 f_{r}, 5 / 3 f_{r}, 7 / 4 f_{r}, 2 f_{r} \\
\quad 3 f_{r}, 1 / 4 f_{r} \text { spectrum series, } 1 / 3 f_{r} \text { spectrum series, } 1 / 2 f_{r} \text { spectrum series, etc. }\end{array}$ \\
\hline \multirow{7}{*}{ Experiment } & $100 \leq \omega<1,000$ & $f_{r}$ \\
\hline & $1,000 \leq \omega \leq 2,910$ & $f_{r}, 2 f_{r}, 3 f_{r}$ \\
\hline & $2,910<\omega<6,070$ & $1 / 2 f_{r}, f_{r}, 3 / 2 f_{r}, 2 f_{r}, 5 / 2 f_{r}, 3 f_{r}$ \\
\hline & $6,070 \leq \omega \leq 6,960$ & $f_{n 1}, f_{r}, 3 / 2 f_{r}, 2 f_{r}, 5 / 2 f_{r}, 3 f_{r}$ \\
\hline & $6,960<\omega<7,980$ & $1 / 2 f_{r}, f_{r}, 3 / 2 f_{r}, 2 f_{r}, 5 / 2 f_{r}, 3 f_{r}$ \\
\hline & $7,980<\omega<8,580$ & $\begin{array}{c}f_{n 1}, 1 / 3 f_{r}, 1 / 2 f_{r}, 2 f_{n 1}, f_{n 2}, f_{r}-f_{n 1}, 2 / 3 f_{r}, f_{r}, f_{r}+f_{n 1}, 4 / 3 f_{r}, 3 / 2 f_{r}, 5 / 3 f_{r}, 2 f_{r}, 3 f_{r}, 1 / 3 f_{r} \\
\text { spectrum series, } 1 / 2 f_{r} \text { spectrum series, etc. }\end{array}$ \\
\hline & $8,580<\omega<10,000$ & $\begin{array}{l}1 / 4 f_{r}, f_{n 1}, 1 / 3 f_{r}, 1 / 2 f_{r}, 2 f_{n 1}, f_{n 2}, f_{r}-f_{n 1}, 2 / 3 f_{r}, 3 / 4 f_{r}, f_{r}, f_{r}+f_{n 1}, 5 / 4 f_{r}, 4 / 3 f_{r}, 3 / 2 f_{r}, 5 / 3 f_{r} \text {, } \\
\quad 7 / 4 f_{r}, 2 f_{r}, 3 f_{r}, 1 / 4 f_{r} \text { spectrum series, } 1 / 3 f_{r} \text { spectrum series, } 1 / 2 f_{r} \text { spectrum series, etc. }\end{array}$ \\
\hline
\end{tabular}

second-order oil film oscillations are delayed, and the peak of the frequency is not obvious in many frequency components [26].

\section{Conclusions}

(1) In the healthy rotor system under the support of the sliding bearing (oil film force), with the speed increasing, the phenomenon of oil film oscillation gradually appears. A series of harmonic components of the frequency division and frequency doubling are very rich. And the frequency division gradually becomes the dominant frequency instead of the rotation frequency. At the same time, the phenomenon of oil film oscillation intensifies.

(2) While the looseness-rubbing coupling fault of rotor system happens, because of the loosing stiffness and rubbing force, the phenomenon of unstable oil film is depressed. The appearance of the first- and second-order oil film oscillation phenomenon is delayed. In the meanwhile, the rubbing phenomenon of the system intensifies and gradually bifurcates from cycle 1 motion to cycle $N$ motion with the speed increasing.

(3) Through studying the dynamics characteristic of the rotor system in different speed condition, according to the research on the dynamics characteristic of the rotor system with different rotational speed, it was found that the looseness-rubbing coupling fault is often characterized by rubbing fault, and the lower part of the time-domain fluctuated shape is denser, while the upper part is relatively loose, and multiple nested half ellipses were shown in orbit diagram. It could be used as a theoretical basis for diagnosing looseness-rubbing coupling fault of rotor-sliding bearing system.
(4) In this paper, the nonlinear dynamic behavior of the coupled fault flexible rotor system supported by the sliding bearing is studied. It is the first time to reveal the relationship between the oil film instability and the rotating speed of the rotor system. It could be found that when the speed reached the certain value, the oil film oscillation occurred. And the frequency of the oil film instability is submerged by the frequency division component caused by the fault. It is not the same as healthy rotor system. And the frequency component of rotor system is dominated by the fault frequency.

\section{Conflicts of Interest}

The authors declare that they have no conflicts of interest.

\section{Acknowledgments}

This work was financially supported by the National Natural Science Foundation of China for Young Scientists (Grant no. 51105065), the Fundamental Research Funds for the Central Universities from Ministry of Education of China (Grant nos. N140304005, N160313003), and National Science Foundation for Postdoctoral Scientists of China (Grant nos. 2014M551105, 2015T80269).

\section{References}

[1] G. Capone, "Orbital motions of rigid symmetric rotor supported on journal bearings," La Meccanica Italiana, vol. 199, pp. 37-46, 1986.

[2] G. Capone, "Analytical description of fluid-dynamic force field in cylindrical journal bearing," L'Energia Elettrica, vol. 3, pp. 105-110, 1991. 
[3] A. Muszynska, "Improvements in lightly loaded rotor/bearing and rotor/seal models," Journal of Vibration, Acoustics, Stress, and Reliability in Design, vol. 110, no. 2, pp. 129-136, 1988.

[4] A. Muszynska and D. E. Bently, "Frequency-swept rotating input perturbation techniques and identification of the fluid force models in rotor/bearing/seal systems and fluid handling machines," Journal of Sound and Vibration, vol. 143, no. 1, pp. 103-124, 1990.

[5] A. Muszynska and D. E. Bently, "Anti-swirl arrangements prevent rotor/seal instability," Journal of Vibration, Acoustics, Stress, and Reliability in Design, vol. 111, no. 2, pp. 156-162, 1989.

[6] H. F. de Castro, K. L. Cavalca, and R. Nordmann, "Whirl and whip instabilities in rotor-bearing system considering a nonlinear force model," Journal of Sound and Vibration, vol. 317, no. 1-2, pp. 273-293, 2008.

[7] J. P. Jing, G. Meng, Y. Sun, and S. B. Xia, "On the oil-whipping of a rotor-bearing system by a continuum model," Applied Mathematical Modelling, vol. 29, no. 5, pp. 461-475, 2005.

[8] G. Adiletta, A. R. Guido, and C. Rossi, "Nonlinear dynamics of a rigid unbalanced rotor in journal bearings. Part I: theoretical analysis," Nonlinear Dynamics, vol. 14, no. 1, pp. 57-87, 1997.

[9] Q. Ding and A. Y. T. Leung, "Numerical and experimental investigations on flexible multi-bearing rotor dynamics," Journal of Vibration and Acoustics, vol. 127, no. 4, pp. 408-415, 2005.

[10] F. Chu and Z. Zhang, "Periodic, quasi-periodic and chaotic vibrations of a rub-impact rotor system supported on oil film bearings," International Journal of Engineering Science, vol. 35, no. 10-11, pp. 963-973, 1997.

[11] C. Liu, H. Yao, Y. Luo, and B. Wen, "Dynamics of rotor-bearing system with coupling faults of pedestal looseness and rubimpact," Zhendong Gongcheng Xuebao, vol. 17, no. 3, pp. 336340, 2004.

[12] G. Chen, "Nonlinear dynamic response analysis of rotor-ball bearing system including unbalance-rubbing-looseness coupled faults," Zhendong yu Chongji, vol. 27, no. 9, pp. 100-104, 2008.

[13] A. Muszynska and P. Goldman, "Chaotic responses of unbalanced rotor/bearing/stator systems with looseness or rubs," Chaos, Solitons \& Fractals, vol. 5, no. 9, pp. 1683-1704, 1995.

[14] C. Q. Su, Y. M. Zhang, and Q. C. Zhao, "Reliability analysis for rubbing in rotor-bearing system with pedestal looseness," Journal of Engineering Design, vol. 15, pp. 347-350, 2008.

[15] Y. Luo, Y. Du, Z. Ren, and B. Wen, "Nonlinear characteristics of two-span rotor-bearing system with coupling faults of pedestal looseness and rub-impact," Transactions of the Chinese Society of Agricultural Machinery, vol. 39, no. 11, pp. 180-206, 2008.

[16] L. Xiang, A. Hu, L. Hou, Y. Xiong, and J. Xing, "Nonlinear coupled dynamics of an asymmetric double-disc rotor-bearing system under rub-impact and oil-film forces," Applied Mathematical Modelling, vol. 40, no. 7-8, pp. 4505-4523, 2016.

[17] A. Hu, L. Hou, and L. Xiang, "Dynamic simulation and experimental study of an asymmetric double-disk rotor-bearing system with rub-impact and oil-film instability," Nonlinear Dynamics, vol. 84, no. 2, pp. 641-659, 2016.

[18] A. Muszynska, Rotor Dynamics, CRC Taylor \& Francis Group, New York, NY, USA, 2005.

[19] H. L. Yao, C. L. Liu, and X. W. Zhang, "Dynamics of pedestal looseness rotor system near the critical speed region," Journal of Northeastern University (Natural Science), vol. 24, pp. 798-801, 2003.
[20] Q. K. Han, T. Yu, and D. Y. Wang, Nonlinear Vibration Analysis And Diagnosis Methods of Fault Rotor System, Science Press, Beijing, China, 2010.

[21] S. Zhou, G. Song, Z. Ren, and B. Wen, "Nonlinear dynamic analysis of coupled gear-rotor-bearing system with the effect of internal and external excitations," Chinese Journal of Mechanical Engineering, vol. 29, no. 2, pp. 281-292, 2016.

[22] N. Lesaffre, J.-J. Sinou, and F. Thouverez, "Contact analysis of a flexible bladed-rotor," European Journal of Mechanics - A/Solids, vol. 26, no. 3, pp. 541-557, 2007.

[23] V. Barzdaitis, M. Bogdevičius, and R. Didžiokas, "Diagnostics procedure for identification of rubs in rotor bearings," Journal of Vibroengineering, vol. 12, no. 4, pp. 552-565, 2010.

[24] J. F. Yang, S. B. Yang, and C. Chen, "Research on sliding bearings and rotor system stability," Journal of Aerospace Power, vol. 23, pp. 1420-1426, 2008.

[25] C. Sun, Y. Chen, and L. Hou, "Steady-state response characteristics of a dual-rotor system induced by rub-impact," Nonlinear Dynamics, vol. 86, no. 1, pp. 91-105, 2016.

[26] C.-C. Wang, "Non-periodic and chaotic response of threemultilobe air bearing system," Applied Mathematical Modelling, vol. 47, pp. 859-871, 2017. 


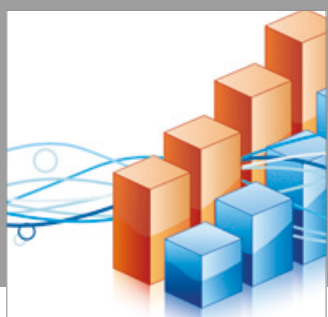

Advances in

Operations Research

vatersals

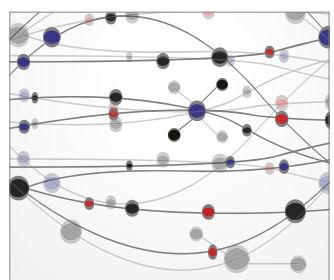

\section{The Scientific} World Journal
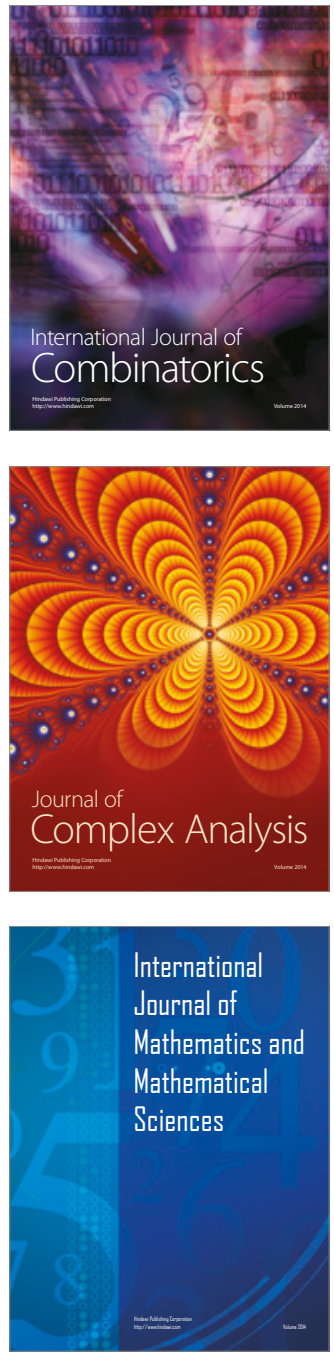
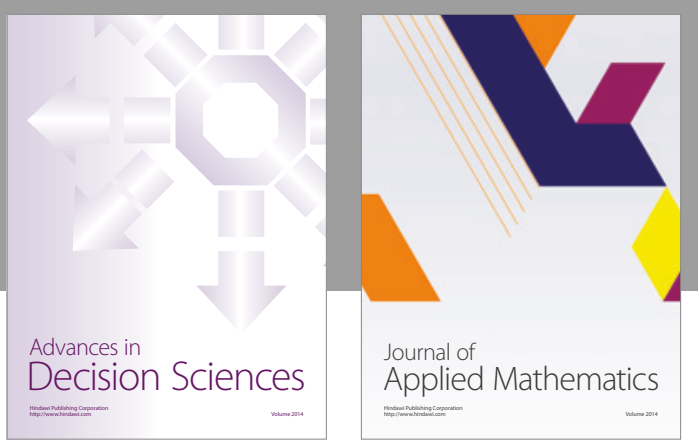

Algebra

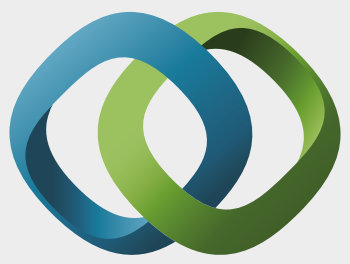

\section{Hindawi}

Submit your manuscripts at

https://www.hindawi.com
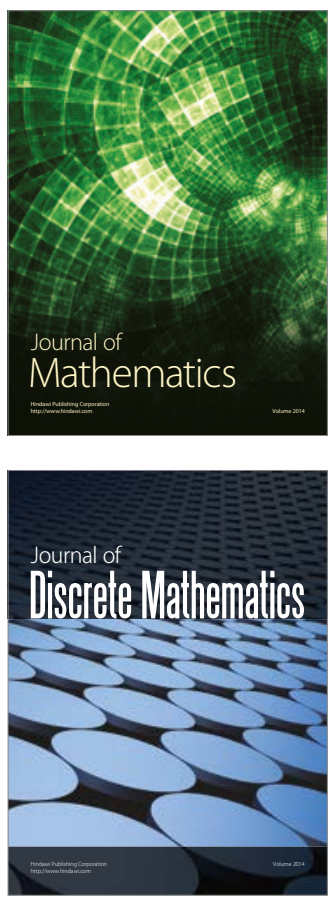

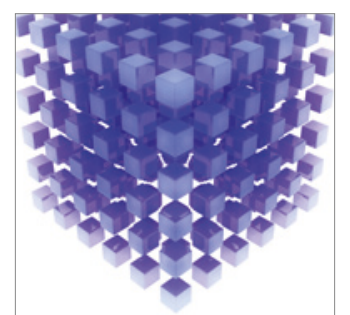

Mathematical Problems in Engineering
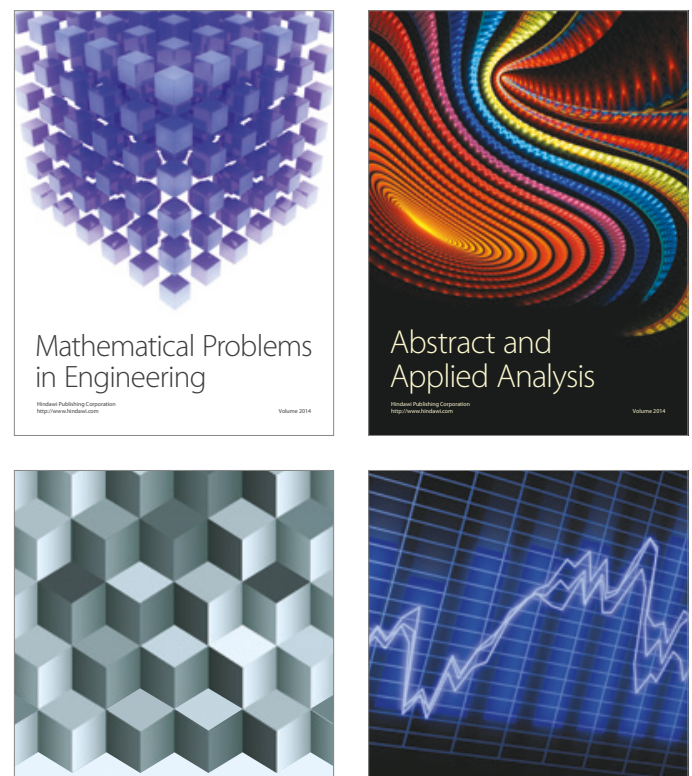

Journal of

Function Spaces

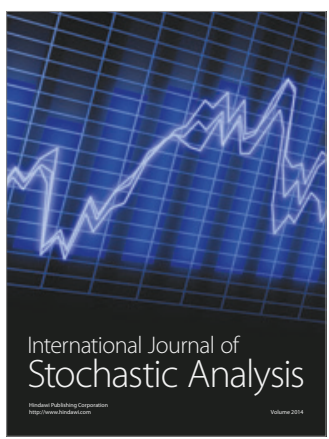

Probability and Statistics
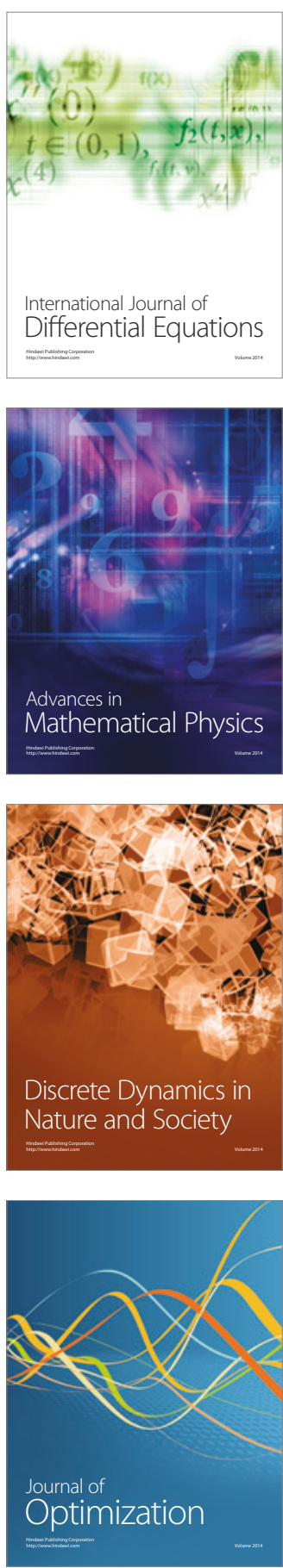